Zur weiteren Prüfung babe ich die oben erwähnten Belichtungsversuche des Herrn Guébhard wiederholt und gefunden, daß auf diese Weise unter Umständen eine Umrißzeichnung erhalten werden kann, aber nur dann, wenn die Maske nicht genïgend fest auf der Platte liegt, derart, daß unter den Rändern der Ausschnitte schmale Halbschattenlinien entstehen, welche bei der Entwicklung nach eintretender Solarisation des Hauptbildes noch normale Schwärzung ergeben. Alle Versuche jedoch, bei denen mit aller Sorgfalt darauf geachtet wurde, daß die Maske (ich wählte galvanisch hergestellte Staniolblätter) allseitig fest gegen die Schicht gedrückt war, ergaben auch bei völliger Solarisation des Hauptbildes keine Spur der Umrißzeichnung. Wenn auch die Insolation mitwirken mag, so kommt ihr mithin jedenfalls zum Zustandekommen der Erscheinung eine durchaus untergeordnete Wirkung zu.

Es bleibt so zur Erklärung der die Umrißzeichnung hervorrufenden schwachen Intensitätsverteilung außerhalb des eigentlichen Bildes genau wie bei der Erklärung der Verbreiterung der Sternscheibchen mit wachsender Expositionsdauer (vergl. Scheiner 1. c.) nur der Hinweis auf die unregelmäßigen Objektivfehler: Rauhigkeit der Flächen, kleine Bläschen und Schlieren. Sie lenken bei dem Strahlendurchgang winzige und für visuelle $Z$ wecke völlig unbedeutende Bruchteile des Lichts vom Bildpunkte ab und rufen eine Lichtverteilung über das ganze Gesichtsfeld hervor, die für das Auge gänzlich unsichtbar ist, aber für photographische Daueraufnahmen merkbar wird. Der Abfall dieser Intensitätsverteilung ist am Bildrande sehr steil (vergl. die von Scheiner gemutmaßte Kurve) und nähert sich später asymptotisch dem Werte Null. Um den Zusammenhang zwischen der Verbreiterung der Sternscheibchen und der photographischen Umrißzeichnung recht deutlich hervortreten $\mathrm{zu}$ lassen, habe ich in meinen oben erwähnten Versuchen mit dem Dreieck zugleich einige künstliche Sterne aufgenommen und an

München, I9I 4 Jan. I 4. passender Stelle deren Aussehen beschrieben. Den bindendsten Grund für die Identität beider Erscheinungen aber sehe ich darin, daß Scheiner für den Radienzuwachs der Sternscheibchen bei beginnender Solarisation des primären Bildchens für 2 ganz verschiedene Instrumente $(34 \mathrm{~cm}$ und $8.2 \mathrm{~cm}$ Öffnung) $0.47 \mathrm{~mm}$ bezw. $0.44 \mathrm{~mm}$ findet, während die Strichbreite an meiner Sonnenaufnahme $0.56 \mathrm{~mm}$ beträgt, also von durchaus derselben Größenordnung ist.

Da nach Messungen von Scheiner das Verhältnis der Intensität, welche die Verbreiterung der Sternscheibchen hervorruft, zur Intensität des Hauptbildes etwa $1 / 10000$ bis $1 / 5000$ ist, so wird zugleich verständlich, warum die photographische Umrißzeichnung auch bei Daueraufnahmen nur in Ausnahmefällen in Erscheinung tritt, und warum im besonderen mein Versuch mit weißen Papierfiguren auf schwarzem Grunde mißlingen mußte: die Helligkeit des »dunklen* Grundes ist für die geringe Intensität der fraglichen Lichtverteilung meist viel zu stark, sodaß bald nach beginnender Solarisation des hellen Bildes auch der Hintergrund solarisiert.

Die bei Aufnahmen sehr heller Objekte beobachtete photographische Umrißzeichnung tritt nach vorstehenden Ausführungen nur ein nach begonnener Solarisation des Hauptbildes infolge starker Uberbelichtung und wird hervorgerufen durch die lichtzerstreuende Wirkung der optischen Teile der Aufnahmeinstrumente. Sie ist ihrem Wesen nach dieselbe Erscheinung, welche wir in der zunehmenden Verbreiterung der Sternscheibchen bei wachsender Exposition seit langem kennen. Umgekehrt kann man die Übereinstimmung beider Erscheinungen, da für die photographische Umrißzeichnung keine andere als die angegebene Ursache erfindlich ist, als Bestätigung der von Scheiner (1. c.) über die Verbreiterung der Sternscheibchen gemachten Äußerungen ansehen.

\title{
Bedeckungen heller Sterne durch den Mond
}

beobachtet am Refraktor (Objektiv Merz $18.6 \mathrm{~cm}$ ) der Düsseldorfer Sternwarte von Dr. W. Luther.

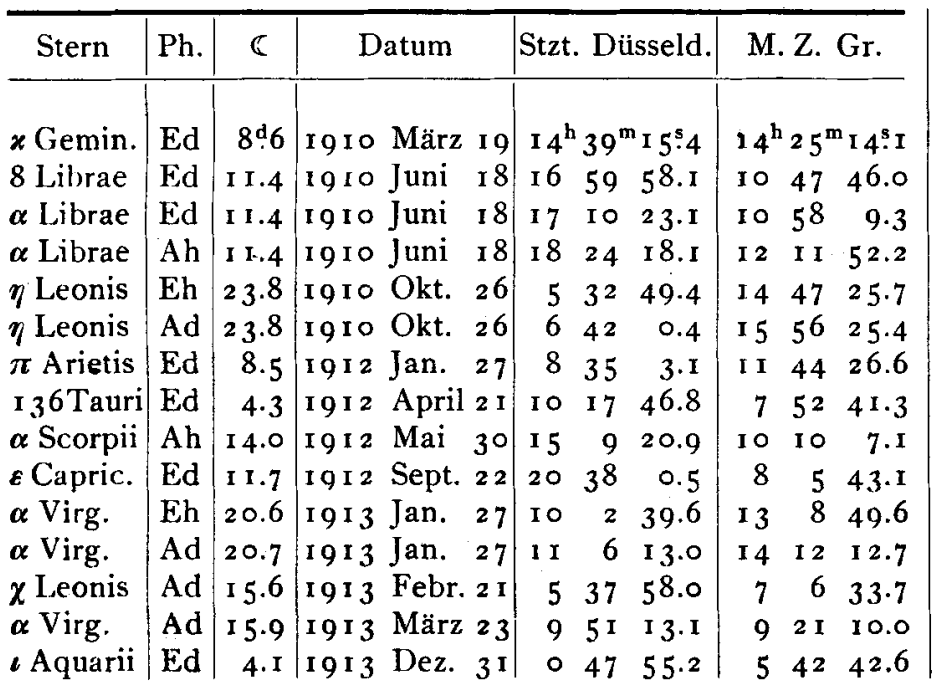

Bemerkungen.

Diese Beobachtungen sind ebenso wie die in A. N. 4396 mitgeteilten nach der Auge-und Ohr-Methode auf der hiesigen städtischen Sternwarte Martinstraße I $\mathrm{I}$ angestellt worden. Die Beobachtungszeiten wurden mit dem inzwischen bekannt gewordenen genauen Wert der Längendifferenz $27^{\mathrm{m}} 2^{\mathrm{s}} .69$ auf $\mathrm{m}$. Zt. Greenwich reduziert.

I9 го März ı 9. Bild des Sterns schlecht infolge tiefen Standes. Eintritt plötzlich. - I9 I0 Juni I8. Eintritt von 8 Librae und $\alpha$ Librae plötzlich am unsichtbaren Mondrand. Austritt von $\alpha$ Librae bei tieferem Stand und Dunst weniger sicher. - r910 Okt. 26. Bei guter Luftbeschaffenheit erschien der von der Sonne nicht beleuchtete Teil des Mondes fast weißgrau. Beim Eintritt war der Stern neben dem blendenden Monde sehr schwach. Der Austritt erfolgte plötzlich. - I9I2 Jan. 27. Der dunkle Rand des Mondes war nicht deutlich erkennbar. Eintritt plötzlich. - I9 I 2 April 2 1. Der von der Sonne nicht beleuchtete Teil des 
Mondes erschien weißgrau. Der Stern klebte am Mondrande, projizierte sich auf den Mond und verschwand dann plötzlich. Vielleicht etwas zu spät. - I 9 I 2 Mai 30. Der Mond war durch Rauch und Dunst stark abgeblendet und erschien matt, der Stern hingegen relativ hell. Der Austritt erfolgte plötzlich. - I 9 I 2 Sept. 22 . Eintritt am unsichtbaren Rande plötzlich. Beim Austritt war der Stern zu schwach. Ich sah denselben im Refraktor erst um $2 \mathrm{I}^{\mathrm{h}} 5^{8 \mathrm{~m}} .8$ Sternzeit, als er -in ähnlicher Entfernung vom Mondrande von nahezu derselben Auffälligkeit war, wie im abgeblendeten Sucher bei der Sonnenfinsternis 19 I 2 April I7 der Lichtpunkt neben der Sonne, über den-ich in A. N. I93.139 berichtet habe. - I9I3
Jan. 27. Der Stern verschwand allmählich. Der Mond stand tief und zwar in einer Dunstschicht. Der durch ein mare gehende Terminator schien nicht auf der Oberfläche zu verlaufen, sondern nach der dunkleren Seite konvex umgebogen tief im Innern des Mondes $z u$ enden, vielleicht infolge glazialer Natur eines solchen mare. Der Austritt erfolgte plötzlich am unsichtbaren Rande. - I913 Febr. 21. Mond tief im Osten. Stern schwach. - I913 März 23. Austritt plötzlich am Nordrande des Mondes. - I 9 I 3 Dez. 3 r. Beobachtung in einer Wolkenlücke. Der Stern verschwand plötzlich, nachdem er etwa 2 Sekunden lang vor dem im Erdlicht sichtbaren Teil des Mondes sichtbar gewesen war.

Düsseldorf, I 9 I 4 Jan. I 5 .

Wilhelm Luther.

\section{Beobachtungen von Algolsternen. Von C. Hoffmeister.}

Im verflossenen Herbst habe ich mit meinem Refraktor von $52 \mathrm{~mm}$ Öffnung, Vergr. 25, einige der helleren Algolsterne nach der Argelandesschen. Methode beobachtet und teile die erlangten Ergebnisse nachstehend mit. Es konnten angeführten waren $U$ Coronae und $U$ Cephei in die Liste der zu beobachtenden Sterne aufgenommen, doch konnte niemals ein Minimum vollständig durchbeobachtet werden. Die Zeiten kleinsten Lichtes für-U Ophiuchi, RS Vulpeculae und U Sagittae sind als vorläufig angenommen $z u$ betrachten und werden nach Ableitung der mittleren Lichtkurven auf Grund weiterer Beobachtungen durch bessere ersetzt werden 3 I Minima an 9 Sternen festgestellt werden. Außer den

können. Sämtliche Zeitangaben der folgenden Tafel sind m. Z. Greenwich. Die 2. Spalte enthält die geozentrischen, die 4. die heliozentrischen Zeiten; $n$ ist die Zahl der Anschlüsse, meist an 2 Sterne. Um eine bessere Abschätzung der Sicherheit der einzelnen Bestimmungen zu ermöglichen, habe ich außerdem in der 6. Spalte die Zeitpunkte für Beginn und Ende der Beobachtungsreihen angegeben. Unter $\mathrm{B}-\mathrm{R}$ finden sich die Vergleichungen mit den Ephemeriden in Prof. Hartwigs Katalog veränderlicher Sterne für I 9 I3. $p$ ist das dem betr. Minimum bei der späteren Ableitung von Mittelwerten für $B-R$ zugeteilte Gewicht.

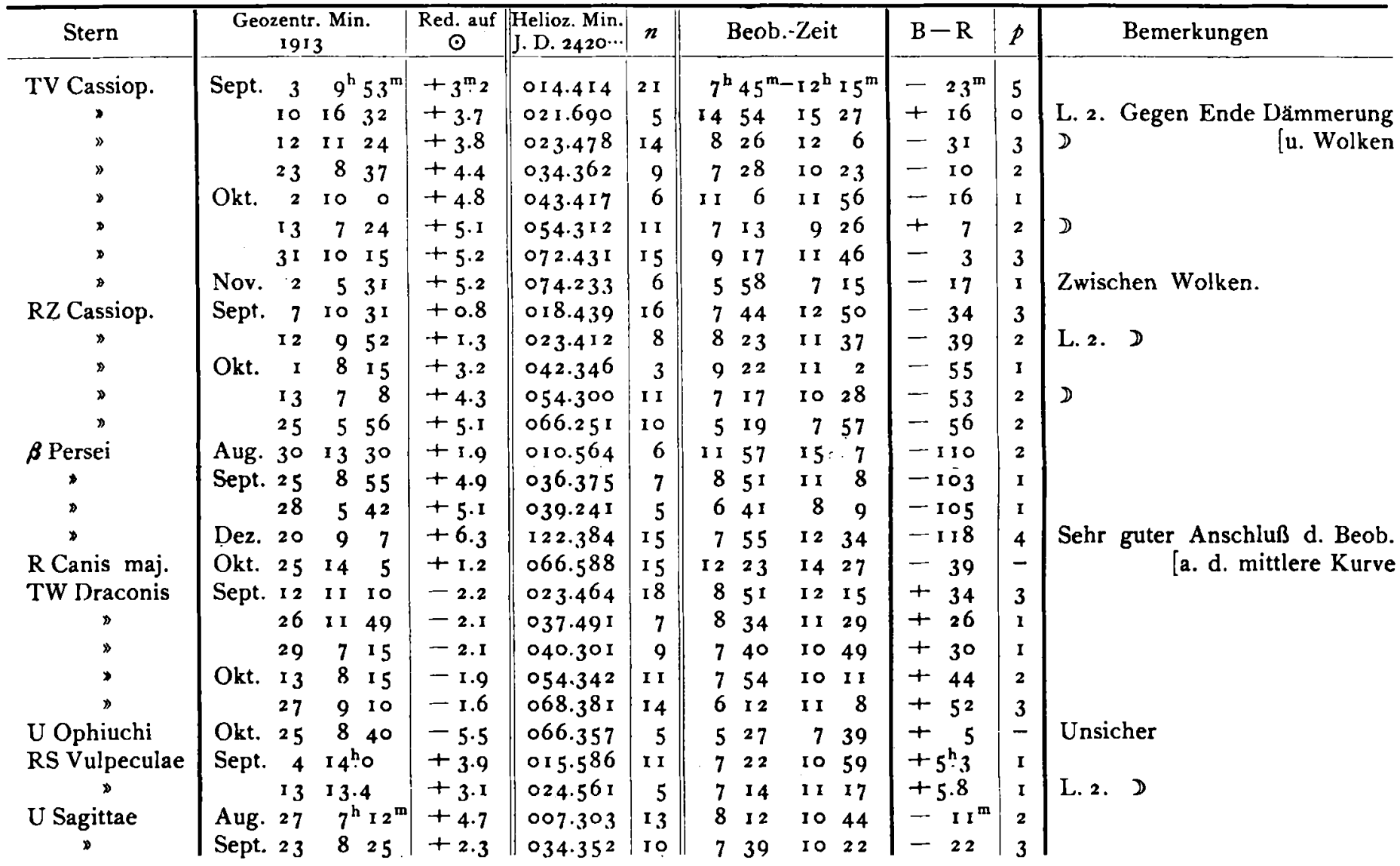

